\title{
Kinematics of Invisible Hinge
}

\section{Toropov A* and Robertis AD}

Dierre S.P.A., Strada Statale per Chieri, Villanova d'Asti, (AT), Italy

\begin{abstract}
Invisible or concealed hinge, having three fixed axes and two slide-able rotational axes, is widely used in the different closures like home doors, laundry machines, cockpit hatches of the boats and even in the notebook computers. The employ of this type of hinge is more advantageous with respect to the traditional surface-mounted hinges in terms of security and aesthetic design.

Unfortunately, due to the lack of knowledge of kinematics of the hinge, the trajectory of the hinge movement is defined empirically in the known designs, and, as a consequence, all these solutions are not optimal. In this paper, we have analyzed the kinematics of five axes hinge, we have obtained the principal analytical relationships between constructive elements and, as a result, we can control the trajectory of hinge by means of following parameters: starting angle between hinge brackets, ratio of the brackets length, and shape of sliding guides. Therefore, this analytical approach can be used as a tool for the design of the concealed hinge which allows having a desirable trajectory of the door movement.
\end{abstract}

Keywords: Invisible hinge; Full opening door; Hinge kinematics

\section{Introduction}

Substantially the present-day configuration of the invisible hinge appears in the patent of Joseph Soss in 1921 and 1924. The facsimile of his original drawings is presented in the Figure 1. As can be seen, this hinge is partly contained inside the thickness of the door and partly inside the thickness of the frame. There are two brackets ( 21 and 22 as specified in the Figure 1), which are connected to rotate about central axis (25). One extremity of the first bracket is connected to the axis fixed on the door. The other extremity connected to the moving axis (27), which is engaged in the sliding guide (19), located on the frame. Another bracket is connected in correspondence with mirror symmetry. In this configuration of the hinge there are 3 fixed axes (one fixed to the door, one central and one fixed to the frame). If there were only these three axes, the trajectory of the door during opening should be vague. Two supplementary axes, moving in the sliding guides, define in unequivocal manner the trajectory of the door. In contrast with one-axis hinge, the center of door rotation moves continuously with increasing the angle of door opening. Initially the central axis is situated internally of the door thickness and finally, when the door is opened, out from the door thickness.

This trajectory allows to open the door up to $180^{\circ}$ and meanwhile don't exhibits the hinge externally, when the door is close.

Another advantage of the invisible hinge is that the doors or openable closures can be hidden in a wall. The term "wall" being used herein in a broad sense which includes side walls, floors and ceiling of building structures, cars, boats etc. Hidden in a wall means that doors, openable panels, and other closures are set with the plane of the face of the panel flush with the plane of the face of the wall.

The recent models of invisible hinge produced by J. Soss factory is shown in the Figure 2. As can be seen the design remains mainly the same as presented in the original drawings. This configuration more or less is repeated by modern producers of hidden hinges for the doors. Main part of commercially known hinges is symmetrical; it means that the door's bracket is equal to the frame's bracket. A typical example some of the recent hidden hinge is shown in the Figure 2.

During the years invisible hinge has been undergoing to the different modifications. One Schmidt [1] is concern of improvement comprises utilization of dense plastic materials having excellent wear and friction reduction characteristics in the slide pins. Some inventors proposed the invisible hinge having asymmetric brackets with different length in order to allow the opening of the door up to $180^{\circ}$ without touch the trim of the jamb or panel of the door leaf Caldari [2]. There are various recent patents, which deal with the adjustment of the invisible hinge in three dimensions Neukoetter and Liermann [3], and Neukoetter [4].

In patents, Way [5] and Liermann [6] are described the hidden hinges, which control the trajectory of the opening the door by means of specially curved sliding paths of the hinge brackets. The invention described in Way [5] may be employed in connection with doors, open-able panels and other closures set with the plane of the face of the panel flush with the plane of the face of the wall. The hinge, which used in this invention, is a 5-axes invisible hinge similar to the Soss hinge, but has a specially curved sliding path. Due to this curved path, the panel in the close position is in the line with the wall, but can be opened and rotate up to the $180^{\circ}$. This hinge has equal brackets of the frame and door leaf parts. The hinge known from Liermann [6] has a sliding guide of the frame part ether extends straight and is positioned at an angle at least $45^{\circ}$ relative to the front surface of the door leaf in the closed position, or arcuate, initially perpendicular and then along to the door leaf in closed position.

All known hinge configurations have been designed empirically, without knowing in what manner effects the changing of one or another constructive parameter on the trajectory of the door, so in this article we determined the principal parameters which are responsible for the

${ }^{*}$ Corresponding author: Alexei Toropov, Dierre S.P.A., Strada Statale per Chier 66/15, 14019 Villanova d'Asti (AT) Italy, Tel: +39 0141 94.94.11; E-mail: alexei.toropov.dierre@gmail.com

Received May 16, 2017; Accepted August 03, 2017; Published August 07, 2017

Citation: Toropov A, Robertis AD (2017) Kinematics of Invisible Hinge. J Appl Mech Eng 6: 281. doi: 10.4172/2168-9873.1000281

Copyright: (c) 2017 Toropov A, et al. This is an open-access article distributed under the terms of the Creative Commons Attribution License, which permits unrestricted use, distribution, and reproduction in any medium, provided the original author and source are credited. 
Feb. 19,1924

$$
\begin{aligned}
& \text { J. SOSS } \\
& \text { HINGE }
\end{aligned}
$$

Filed Nov, 21, 192
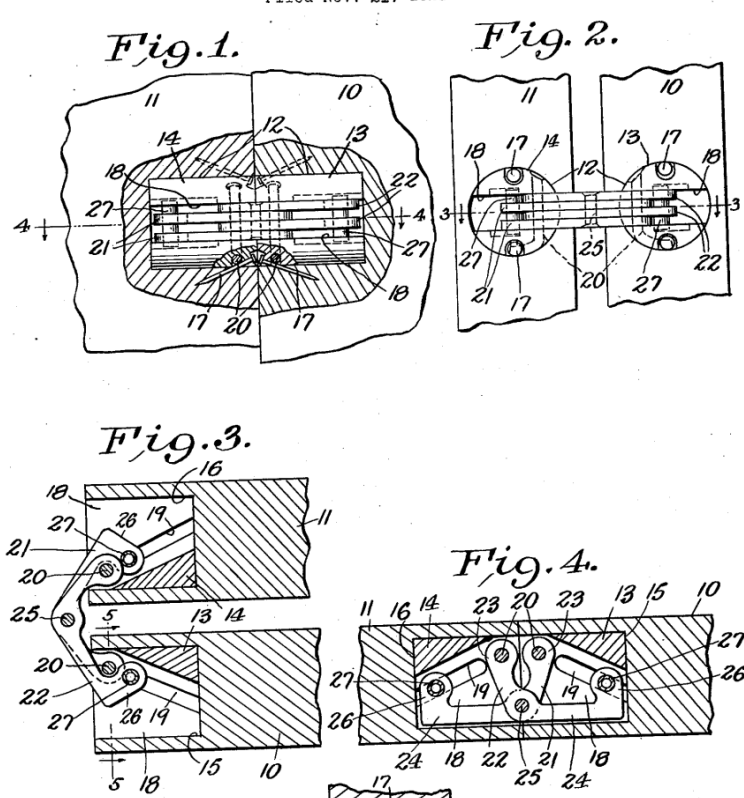

\section{Fig. 4 .}
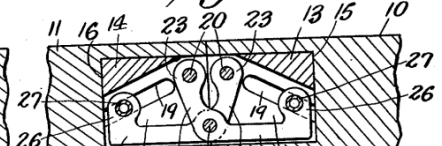

$241822121 / 18$
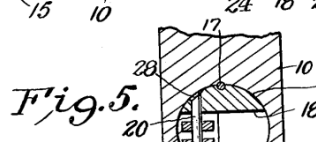

${ }_{20}, \mathrm{t}$
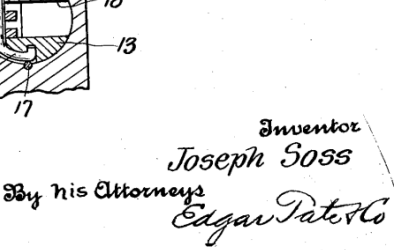

Figure 1: Drawings of invisible hinge invented by Joseph Soss in 1924 publication number US1484093 A [1].

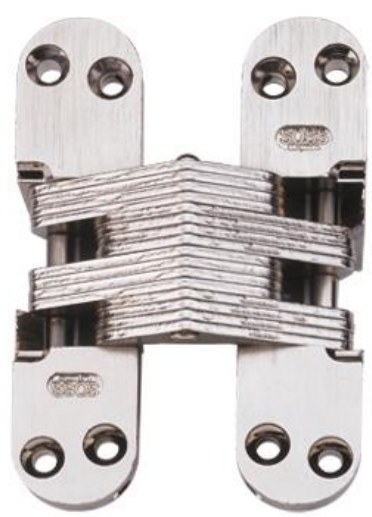

Figure 2: An example of recent invisible hinge with five axis motions

hinge movements and obtain the analytical equations for calculation of the closure trajectories.

\section{Trigonometric Analysis of the Motion of the Invisible Hinge with 5 Axes}

Figure 3 presents the geometry of layout of invisible hinge having 5 axes. This is schematically shown a transverse section of the hinge, mounted in hidden position, partially in the door frame and partially in the door leaf. It looks as a scissors with two brackets which are connected to each other in central axis. One end of the first bracket is hinged on the axis, fixed on the frame, but another end is engaged in the sliding guide of the door leaf. In its turn, one end of the second bracket hinged on the axis, fixed on the door leaf and another end correspondingly engaged in the sliding guide of the frame.

One of the principal parameters, which control the motion of the hinge, is an angle $\alpha$ of the "scissors". The motion of the hinge elements initiated from the initial angle $\alpha_{\text {initial }}$ to the final angle $\alpha_{\text {final }}$, which have lower value than initial angle and can be also equal to zero. Let we name the brackets belong to the door leaf $r_{1}$ and $R_{1}$, when $r_{1}$ is the length of the short bracket of the door leaf (equal to the distance from central axis to the axis fixed on the door leaf), and $R_{1}$ is the length of the long bracket of the door leaf and $r_{2}$ e $R_{2}$ the short and long brackets of the frame correspondingly, as shown in the Figure 3. In this scheme is shown the general case, that is all of these four brackets can have any length, but with the following constrains: $r_{1}<R_{1}$, and $r_{2}<R_{2}$, otherwise it will change the direction of rotation [7-10].

To simplify the hinge movement description, let us consider only the part of the hinge, which belongs to the door, because the behavior of the other part (belong to the frame) is identical with respect to central axis of hinge (point $\mathrm{A}$ on the Figure 4). It means that the trajectories of the door and the frame are similar when the origin of Cartesian coordinate system is placed in the central axis of the hinge.

Let us draw the circle around the axis fixed on the door leaf (point B) with the radius equal to the difference between the lengths of the brackets R-r (Figure 4). Then we draw from the sliding axis (point D on the Figure 4) two beams tangent to this circle: DF and DG. So, as can be seen, all geometrically allowable sliding guides are included in the area between these two beams. Let us suppose that initial angle of this "scissors" is $\alpha_{0}$. The final angle is 0 . When the "scissors" are closing, the sliding axis (the extreme point $\mathrm{D}$ of the long bracket) slips along the sliding guide DF (sliding guide of "high rotation") and, as a consequence, the door will rotate. As can be seen on the Figure 4 the leaf door rotates on the angle $\beta$ in this case. If the axis $\mathrm{D}$ will run along the beam DL ("moderate rotation"), when angle $\alpha \rightarrow 0$ the door leaf swings to the angle $\theta$. And finally, if the axis $\mathrm{D}$ slides along the line DG, the door leaf rotates on the very low angle $\zeta$. So we have determine the geometry of sliding guides, which provide the high, medium and low rotation of the door leaf when the two brackets of the "scissors" are closing (when the angle $\alpha$ go from $\alpha_{0}$ to 0 ): "high rotation" along the line NS, "moderate rotation" along the line NP and "low rotation" along the line NM.

As can be seen from Figure 4 the angle of high rotation $\beta$ is equal to:

$$
\beta=2 \times \omega+\zeta=\alpha / 2+\gamma+\omega
$$

Where $\omega$ is the angle between two beams $\mathrm{BF}$ e $\mathrm{BD}$ of the orthogonal triangle BFD, which is equal to the orthogonal triangle BGD, and $\zeta$ is the low rotation angle and is the angle between the beam BG and horizontal line (Figure 4).

The angle of moderate rotation can be represented in terms of $\gamma$ and $\alpha$ from the triangle ALD:

$$
\Theta=\pi+(\pi+\gamma-\alpha / 2)=\alpha / 2+\gamma
$$

Finally, the angle of the low rotation can be expressed as (look to the beam $A B$ on Figure 4 ): 


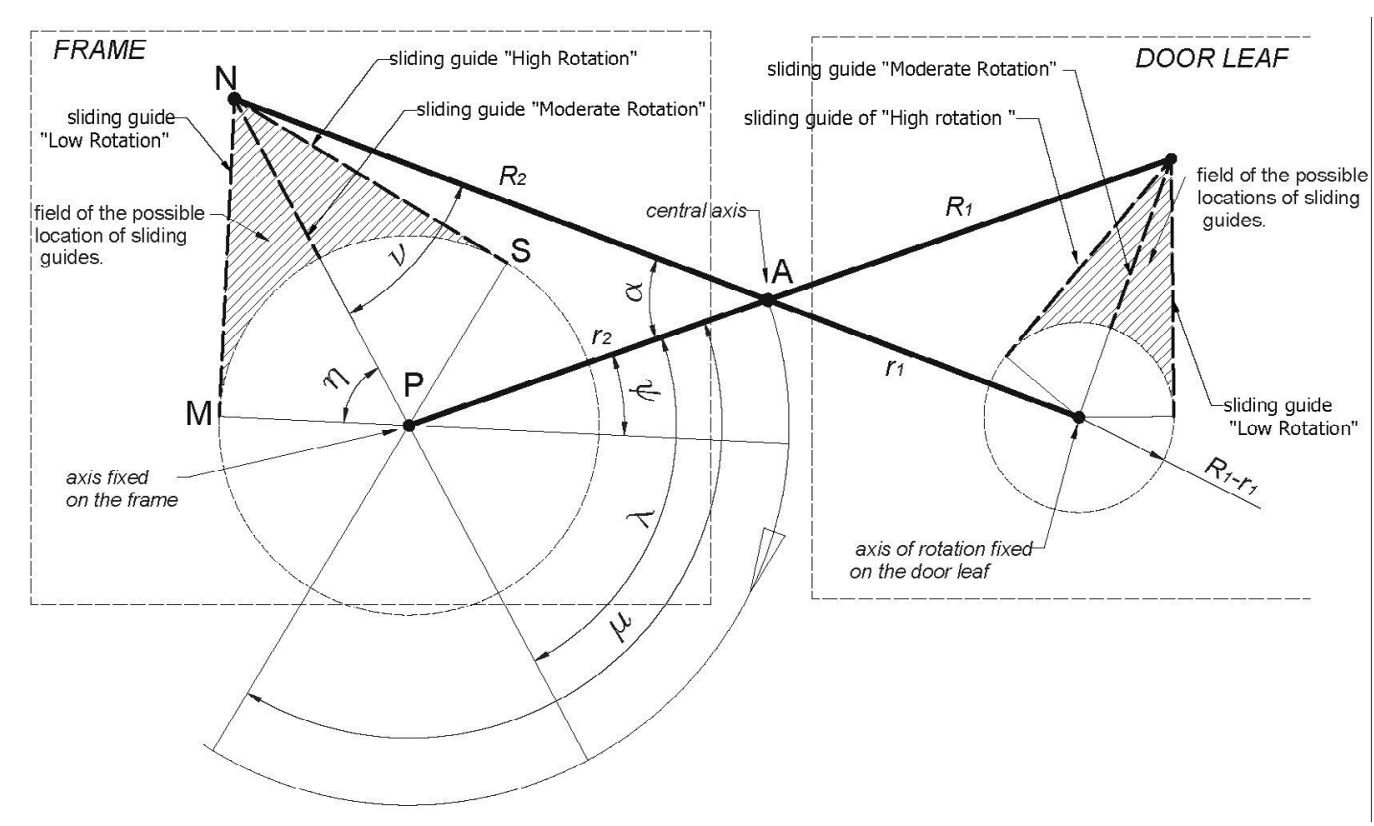

Figure 3: Schematic presentation of the 5-axis invisible hinge.

A

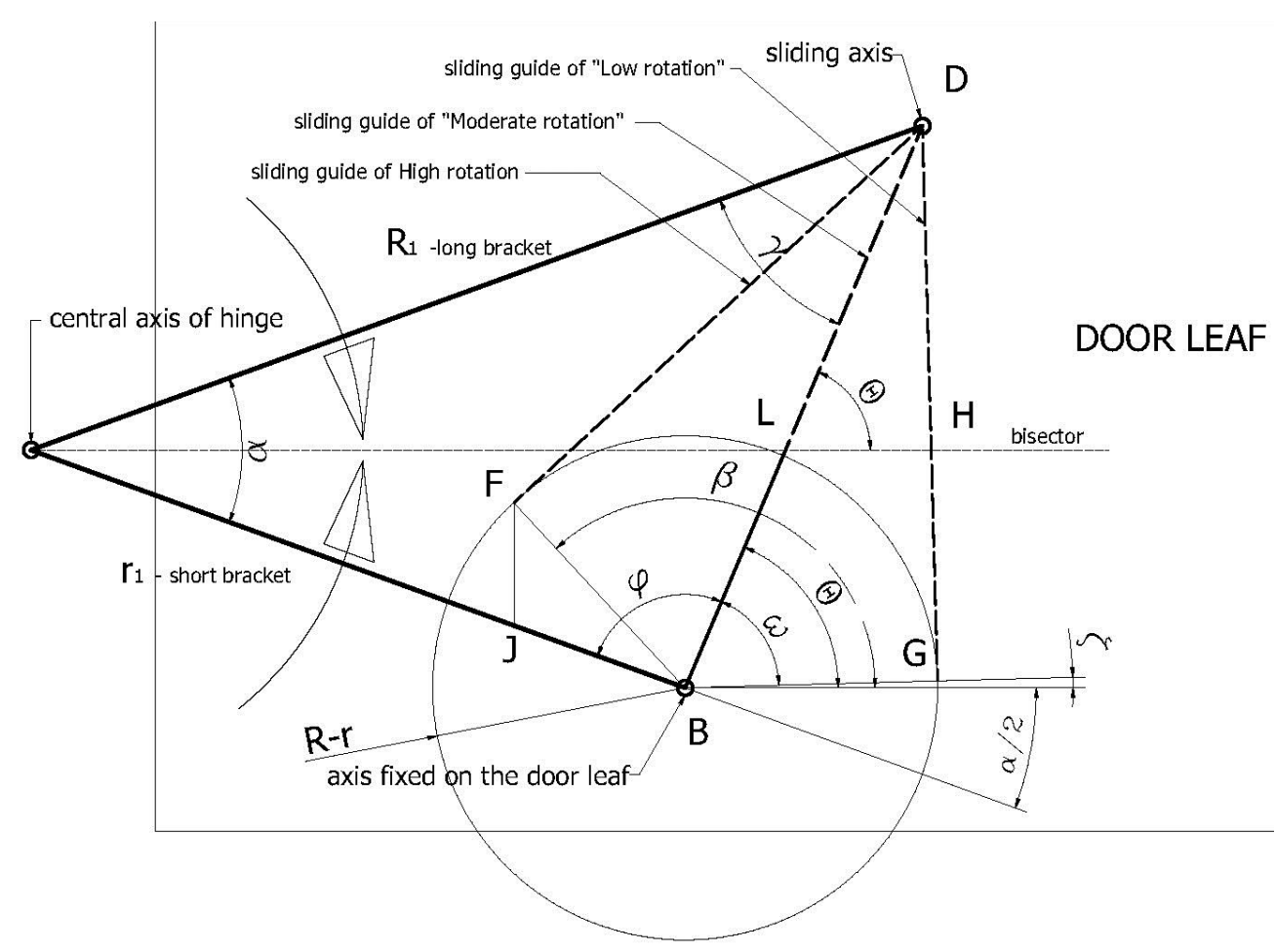

Figure 4: The part of invisible hinge which belongs to the door in the coordinate system fixed on the central axis of the hinge (point $A$ is a centre of coordinate).

$$
\zeta=\pi-\alpha / 2+\phi-\omega=\alpha / 2+\gamma-\omega
$$

Where the angle is derived from the triangle ADB: $\phi=\pi-\alpha-\gamma$

The angle is a varying argument when the hinge moves, so in order to obtain the rotation trajectory of the door leaf as a function of the constructive parameters of the hinge we should find the angles $\gamma$ and $\omega$.
Let us consider the triangle ADB created between two brackets. In order to find the angle $\gamma$ it can be used the theorem of sinus:

$$
\operatorname{Sin}(\alpha) / \mathrm{r}=\sin (\gamma) / \mathrm{c}
$$

Where $\mathrm{c}$ is the length of opposite beam $\mathrm{BD}$ to the angle $\langle$ in the triangle $\mathrm{ADB}$, so it can be expressed as [8]: 


$$
\mathrm{c}=\left[\mathrm{r}^{2}+\mathrm{R}^{2}-2 \times \mathrm{r} \times \mathrm{R} \times \cos (\alpha)\right]^{1 / 2}
$$

Then we define the geometrical parameter of the hinge $\mathrm{k}$, as a ratio of the short bracket to the difference between long and short brackets (radius of the circle on the Figure 4), so $k=r /(R-r)$.

From (4) e (5) we can obtain the angle $\gamma$, as a function of two arguments $\alpha$ and $\mathrm{k}$ :

$$
\gamma=\arcsin [\mathrm{k} \times \sin (\alpha) / \mathrm{g}(\mathrm{k}, \alpha)]
$$

Where $g(k, \alpha)=\left[k^{2}+(1+k)^{2}-2 \times(1+k) \times k \times \cos (\alpha)\right]^{1 / 2}$ is a function of $\alpha$ and $\mathrm{k}$.

In order to find the angle $\omega$ we look on the triangle BGD, this is an orthogonal triangle, thus $\cos \omega=(\mathrm{R}-\mathrm{r}) / \mathrm{c}$, and taken (5) into consideration we obtain:

$$
\omega=\arccos \left[\mathrm{g}^{-1}(\mathrm{k}, \alpha)\right]
$$

Finally we can express all above defined rotation angles of the door leaf with respect to the central axis in terms angle between brackets and the ratio of the bracket lengths $k=r /(R-r)$.

The graph on the Figure 5 represents two angles of rotation of the door leaf as a function of brackets length ratio " $k$ " with respect to the central axis of hinge A. It has been calculated by using the equations (1), (2) and (4)-(7). This calculation shows that if the angle between brackets of the "scissors" is closed from $60^{\circ}$ to $10^{\circ}$, so the angle difference is only $50^{\circ}$, but at the same time the door leaf can rotate up to $\approx 93^{\circ}$, in correspondence of the sliding guide trajectory. As can be seen, the maximum value of the rotation is achieved at the $\mathrm{k}=2$, that is $\mathrm{R} / \mathrm{r}=3 / 2$, and then slowly decreases, when R gradually is reaching the $\mathrm{r}$.

Figure 3 show the overall structure of five - axis hinge, as can be seen, the part belong to the frame is mirror symmetrical with respect to the vertical axis which cross the central axis (assuming, obviously, that the length of the brackets is the same). Therefore, it reasonably to suppose that the rotation of the "frame" part of the invisible hinge can be expressed in the same manner and correspondent rotation angles are the same function of the central angle $\left\langle\right.$ and the ratio $k_{2}=r_{2} /\left(R_{2}-r_{2}\right)$.
Where the index " 2 " refers to the frame, while " 1 " one - to the door leaf (see the designations on the Figure 3 ).

In the above consideration, the central axis was a fixed reference point, but in the real situation the central point moves, and only one point of the hinge is fixed - the joint $\mathrm{P}$ on the frame (Figure 3). Due to the inertial coordinate system, the door trajectory will be the sum of the door rotation with respect to the central axis and the motion of central axis with respect to the point $\mathrm{P}$, fixed on the frame. As can be seen on the Figure 3, we consider, as for the door leaf, there are three possible sliding guides on the part of the hinge, belong to the frame. There are: "high rotation" along the line NS, "moderate rotation" along the line NP and "low rotation" along the line NM. Therefore, when the hinge is opening, the axis $\mathrm{N}$ slides along the guide $\mathrm{NM}$, and the central angle a goes to 0 , the central axis (point $\mathrm{A}$ ) rotate around the fixed joint $\mathrm{P}$ up to the angle equal to $\psi$, as shown on the Figure 3. Similarly, the central axis rotate around the joint $\mathrm{P}$ for the angle " $\lambda$ " if axis $\mathrm{N}$ slides up to the guide NP ("moderate rotation") and for the angle $\mu$ when the sliding guide is NM ("high rotation").

All these three angles of rotation can be expressed in terms of three constructive parameters of the hinge: initial central angle $\alpha$ and the lengths of the brackets $r_{2}$ and $R_{2}$. As can be derived from the triangles APN and NPM on the Figure 3:

$\psi=\alpha+v-\eta ; \lambda=\alpha+v ; \quad \mu=\alpha+v+\eta ;$

Where: $\left(\mathrm{R}_{2}-\mathrm{r}_{2}\right)$

$v=\arcsin \left[\mathrm{k}_{2} \times \sin (\alpha) / \mathrm{g}\left(\mathrm{k}_{2}, \alpha\right)\right], \eta=\operatorname{arc} \cos \left[\mathrm{g}^{-1}\left(\mathrm{k}_{2}, \alpha\right)\right], \mathrm{k}_{2}=\mathrm{r}_{2} /$$$
\text { and } \mathrm{g}\left(\mathrm{k}_{2}, \alpha\right)=\left[\mathrm{k}_{2}^{2}+\left(1+\mathrm{k}_{2}\right)^{2}-2 \times\left(1+\mathrm{k}_{2}\right) \times \mathrm{k}_{2} \times \cos (\alpha)\right]^{1 / 2} \text {. }
$$

As can be seen from the Figure 3, the angles $\psi, \lambda$ and $\mu$ are the angles of rotation around point $\mathrm{P}$ of the bracket PA, but we need obtain the angles of rotation of the hinge bisector, because the angles of rotation of the door leaf (1), (2) and (3) have been calculated around point A with fixed orientation of the bisector (Figure 4). So, to obtain the angles of rotation of bisector we need to subtract,

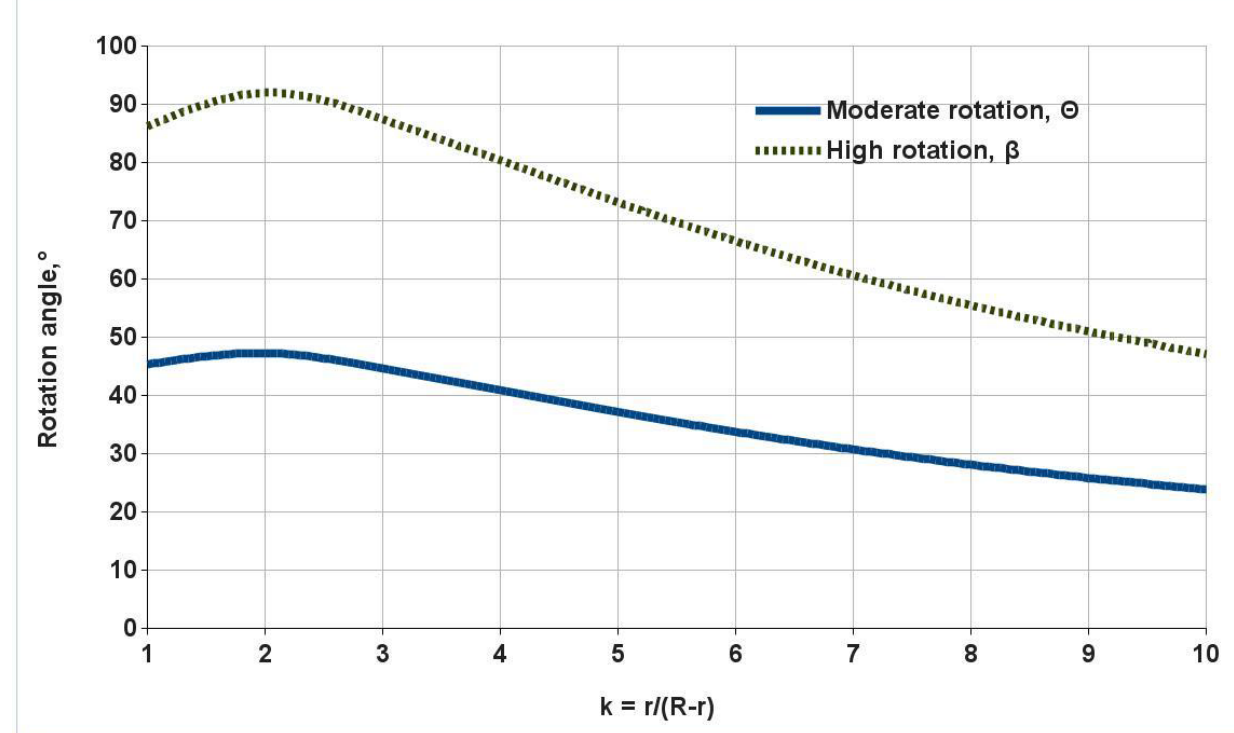

Figure 5: Angle of rotation of the door leaf with respect to the central axis A vs. k for two sliding guides trajectories: "moderate rotation" and "high rotation", (Figure 4). Starting angle $\alpha=60^{\circ}$, final angle $\alpha=10^{\circ}$. 
The half angle between brackets $(\alpha / 2)$ from the $\psi, \lambda$ and $\mu$ :

$$
\psi^{\prime}=\alpha / 2+v-\eta ; \lambda^{\prime}=\alpha / 2+v ; \quad \mu^{\prime}=\alpha / 2+v+\eta ;
$$

Where, $\psi^{\prime}, \lambda^{\prime}$ and $\mu^{\prime}$ are the angles of rotation for different sliding guides: "low rotation" along the line NM, "moderate rotation" along the line NP and "high rotation" along the line NS, correspondingly, when a go up to 0 . As can be seen, the expressions for the angle of rotation of the bisector around the point $\mathrm{P}$ are similar to the expressions of the rotation of the door leaf with respect to the central point A.

Let us locate the reference point of the Cartesian coordinate system to the frame and calculate overall moving of the door leaf. In order to obtain the overall rotation of the door leaf with respect to the frame, we should to sum the two rotations: door leaf with respect to point $A$ and bisector of the hinge around the point $\mathrm{P}$, fixed on the frame.

\section{Discussion and Validation}

Figure 6 presents the whole rotation of the door leaf with respect to the frame when the trajectories of sliding guides, as for the door leaf, as for the frame are both the trajectories of "high rotation", Figures 3 and 4. In the other words, in this case the angle of the door leaf rotation is equal the sum of the angles $\left.\mu^{\prime}+\right\rangle \beta$ from the equations (1) and (9). As can be seen, in the case of the specific trajectories and certain ratios of a brackets length, the changing in the angle $\alpha$ between the brackets only for $\approx 45^{\circ}$ allows reaching up to $180^{\circ}$ of the rotation of the door leaf. As for the fixed central axis, also in this case the maximum value of the rotation is achieved at the $\mathrm{k}=2$, that is at the brackets length ratio $\mathrm{R} / \mathrm{r}=3 / 2$.

Figure 7 show the angle of the door leaf rotation with respect to the door frame as a function of the angle between two brackets for different trajectories of sliding guides as for the door part of the hinge, as for the frame part. As can be seen, the maximum angles are achieved when both trajectories of sliding guides are of "high rotation", Figure 3. In this case the brackets length ratio is $\mathrm{k}=3$ for both parts of the hinge. It means that the configuration of the hinge can be as symmetrical and asymmetrical with respect to the central axis, but only one constraint imposted: the brackets ratio $\mathrm{k}$ is equal to 3 . Also the graph show that for the "high rotation" guide trajectory, when the angle between brackets changed for $80^{\circ}$ (that is from $80^{\circ}$ to $0^{\circ}$ ), the door leaf can be done one complete revolution, that is it rotates up to $360^{\circ}$. However, the same changing of the angle a between the brackets causes the twist of the door leaf less than $50^{\circ}$ when the both trajectories of the sliding guides are of "low rotation". It means that by changing the trajectories of sliding guides and the ratios of brackets of the hinge, it's possible to control the trajectory of the door leaf. For example, let us consider the following configuration of the hinge: the door part of the hinge going along the sliding guide of "low rotation", but the frame part of the hinge slips along the guide of "high rotation". In this case the door practically doesn't rotate with respect to the bisector of the angle " $\alpha$ ", but all rotation occurs around the axis P, fixed on the frame (Figure 3). Thus the initial movement of the door transforms in practically linear displacement. A good indicator of the type of trajectory is the derivative of the angles " $\beta$ " and " $\mu$ " with respect to the angle between brackets $(\alpha)$. So, low value of derivative $d(+\mu) / d \alpha$ means the low twist and almost linear displacement and differently, high values of derivative $d(\beta+\mu) /$ $\mathrm{d} \alpha$ indicates practically poor rotation.

Also it possible to modify the door trajectory by means of a curving of the sliding guides. For example, initial part of the sliding guide can be of "high rotation", but the final part can be "low rotation", changing in this manner the trajectory of the door during it opening. This modification of sliding guide pathway can be implemented as for the door part of the hinge, as well for the frame part, enhance thereby the possible variants of trajectories of the door leaf movement during opening.

The above trigonometric analysis has been verified by creation the CAD model of the hinge with arbitrary chosen configuration. The model is presented in the Figure 8. As can be seen, the model represents the trajectory of hinge components during the procedure of door opening. In this example we have chosen the sliding guides, as for frame part, as for door part, both of "high rotation". In the Figure 8 are shown also the corresponding brackets lengths and parameter $\mathrm{k}$ for door leaf and frame parts. There are presented a three consecutive positions of the hinge elements and the door leaf during the opening: first, the initial

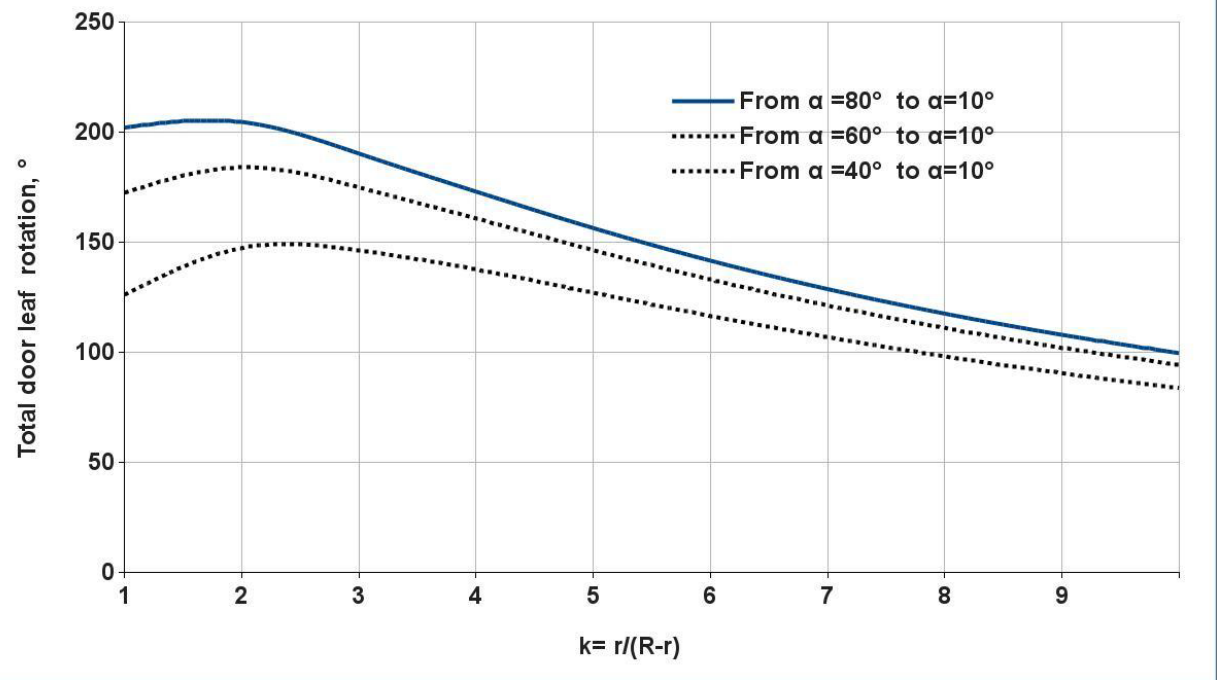

Figure 6: Total rotation of the door leaf with respect to the axis $\mathrm{P}$ of frame as a function of $\mathrm{k}$ for sliding guide of "high rotation" and for three starting angles $\alpha=80^{\circ}$, $60^{\circ}$ and $40^{\circ}$ and final angle $\alpha=10^{\circ}$. In this example we assume the same ratio of the brackets for door and the frame parts of the hinge, that is $k_{1}=k_{2}$. 


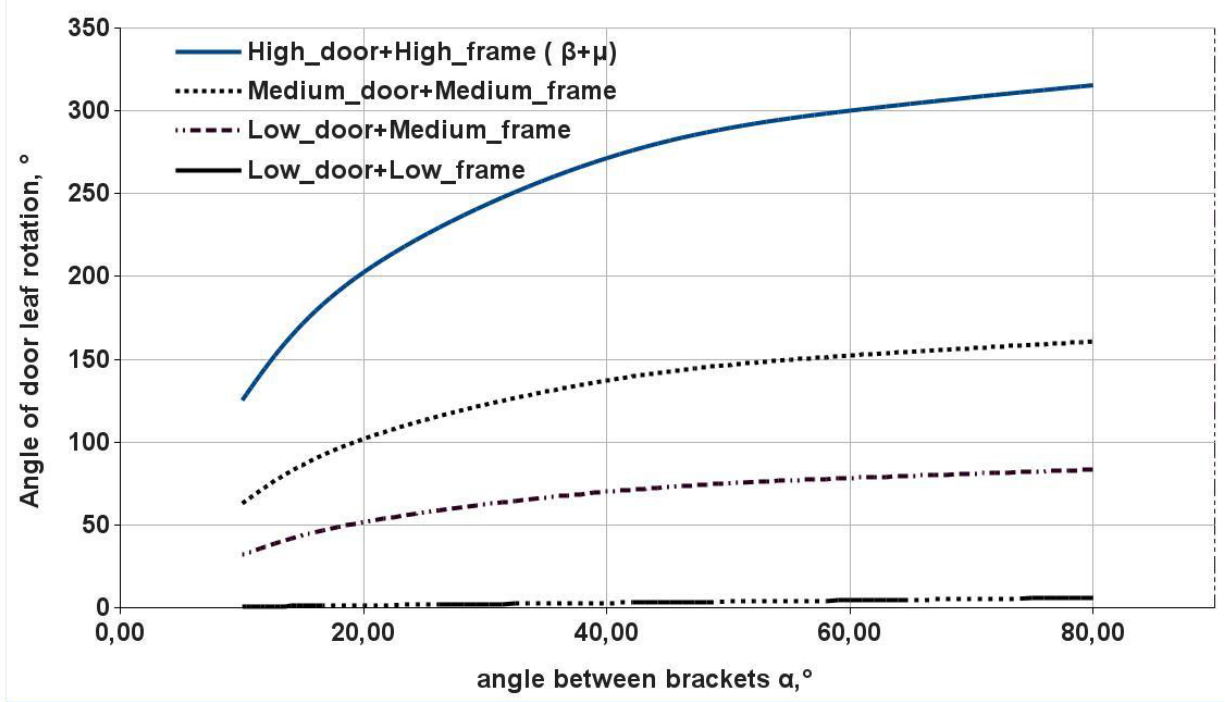

Figure 7: Total rotation of symmetrical hinge layout $\left(\mathrm{k}_{1}=\mathrm{k}_{2}\right)$ as a function of starting angle of brackets $\alpha$ (the final angle always 0 ). Four graphs show the angle of door rotation for different trajectories of sliding guides at a fixed brackets ratio $\mathrm{k}=3$.

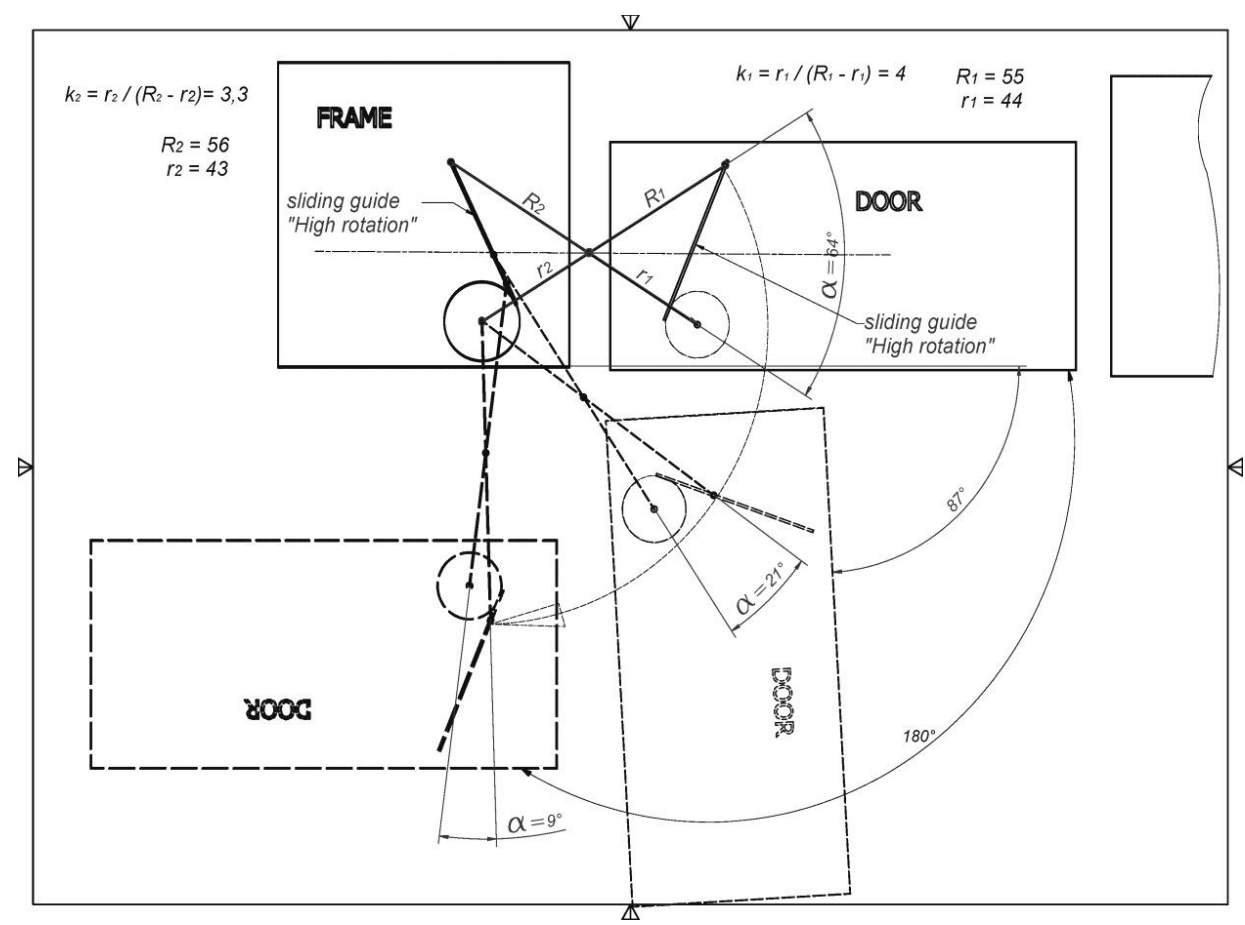

Figure 8: An example of the 5-axis hinge configuration with arbitrarily chosen specific dimensions of brackets in three consecutive positions for verifying the present trigonometric analysis.

angle between brackets is equal to $64^{\circ}$ door rotation angle is 0 , in the second position the angle $\alpha=21^{\circ}$, and the door is rotate up to $87^{\circ}$, and in the final position the angle $\alpha=9^{\circ}$, and the door rotation angle is equal to $180^{\circ}$. All these angles have been obtained from the CAD model of the hinge (Figure 8). If the presented above trigonometric analysis is correct we must achieve the same rotation angles of the door by using the following parameters of the hinge configuration: four values of the brackets lengths (which transform in the values of $\mathrm{k}_{1} \mathrm{e}_{2}$ ) and the value of angle between brackets. Inserting these parameters in the equations for $\beta$, equation (1) and for $\mu^{\prime}$ equation (9), we can calculate the angle of the leaf door rotation for above three positions:

Second position on the Figure 8: $\Delta \beta=\beta\left(\mathrm{k}_{1}, 64^{\circ}\right)-\beta\left(\mathrm{k}_{1}, 21^{\circ}\right)=$ $40,5^{\circ}$ is the angle of rotation of the door leaf with respect to the bisector of $\alpha$; $D \mu^{\prime}=\mu^{\prime}\left(\mathrm{k}_{2}, 64^{\circ}\right)-\mu^{\prime}\left(\mathrm{k}_{2}, 21^{\circ}\right)=45,4^{\circ}$ is the angle of rotation of the bisector line around the axis $\mathrm{P}$, fixed on the frame.

And summarizing these angles, we obtain the angle of the door leaf rotation with respect to the frame: $\mathrm{Db}+\mathrm{D} \mu^{\prime}=85.6^{\circ}$. 
Correspondingly for third position on the Figure 8 we have : $\Delta \beta$ $=\beta\left(\mathrm{k}_{1}, 64^{\circ}\right)-\beta\left(\mathrm{k}_{1}, 9^{\circ}\right)=87,6^{\circ}$ - rotation of the door leaf with respect to the bisector; $\mathrm{D} \mu^{\prime}=\mu^{\prime}\left(\mathrm{k}_{2}, 64^{\circ}\right)-\mu^{\prime}\left(\mathrm{k}_{2}, 9^{\circ}\right)=92,5^{\circ}$ - rotation of the bisector around the axis $\mathrm{P}$. The sum of these angles result in the door leaf rotation: $\mathrm{Db}+\mathrm{D} \mu^{\prime}=180.1^{\circ}$.

As can be seen the calculated values are in a good accordance with the real modeling, in fact: in second position the angle of rotation is $85,6^{\circ}$ in comparison with $87^{\circ}$, and in third position the rotation angle is equal to $180,1^{\circ}$ in comparison with $180^{\circ}$ for CAD model. Therefore, the presented analysis is correct and gives the possibility to evidence the specific parameters, which determine the trajectory of the hinge during opening the door. There are: the ratio of the brackets lengths of the door part " $\mathrm{k}_{1}$ " and the frame part " $\mathrm{k}_{2}$ ", the angle between brackets " $a$ ", and sliding pathways for both parts of the hinge. At that, we have determined the area where the sliding pathways can exist and have defined the trajectories of sliding guides which can result in high or low values of the changing in the angle of door rotation in relation to the increment of the angle between brackets.

It should be noted, that a direct CAD computation of the hinge movement by means of increment or decrement of the above variables (lengths of brackets, angle between brackets and multiplicity of sliding trajectories) has a billions of different solutions. It makes impossible any valid consideration about behavior of the hinge as a function of these constructive variables. In contrary, an analytical approach, described in this article, gives the possibility to understand the influence of different parameters and gives the answer to question, how to construct one or another required trajectory of the door leaf.

\section{Analysis of known configurations of the concealed hinge with 5 axes}

We can analyze a different known hinge from the point of view of the above analytical approach.

The most widely employed type of concealed hinge has a symmetrical configuration, very close to that, firstly proposed by Joseph Soss in 1921. The original drawings are presented in the Figure 1, and the modern appearance is shown in the Figure 2. Taken the measurements from drawing 4 in the Figure 1, we obtain approximately $\mathrm{k}=\mathrm{r} /(\mathrm{R}-\mathrm{r})=5.3$, and $\alpha=50^{\circ}$, but in the closed position the long bracket line overlap the short bracket up to approximately $8^{\circ}$, so the angle span is equal to approximately $60^{\circ}$. If we insert these values in the equations (2) e (6), supposing, as can be recover from the Figure 1, that the hinge has a sliding guide of "moderate rotation", we obtain $81.5^{\circ} \times 2=163^{\circ}$ for the whole angle of the door rotation, instead of $180^{\circ}$. But observed carefully these antique drawings it can be seen that the sliding guides slightly inclined to the direction of "high rotation". This can explain this small discrepancy. Anyway, this sliding pathway is not optimal for the symmetric hinge and can be modified by using for example the "high rotation" location of sliding guides.

Hinge configuration known from Liermann, [8] both sliding guides are straight and have a position of "high rotation". As a consequence, the span of the angle between brackets (a range of angle a from closed to completely open door position) is low, namely at about $35^{\circ}$ for frame part, and $45^{\circ}$ for leaf door part. This result in also, that the central axis came out from the frame plain just at the angle of door rotation at about $90^{\circ}$, while for the hinge with sliding guide of "medium rotation" this happens only at the angle of $180^{\circ}$. The drawback of this arrangement is the high offset in the direction normal to the door plain between the frame part and door part of the hinge. But this offset can be easily minimized using the kinematic analysis presented above.
In some recent hinge assemblies, it was implemented the curved sliding guides. For example the sliding guide of frame part of the door hinge, which is known from Liermann [6], has a shape approximately of circular arc. But the most part of this sliding guide pass along the direction of "low rotation", as is defined in this article and shown on the Figure 3. While the sliding guide of the door leaf part is straight and correspondent approximately to the position of "high rotation", as also shown in the Figure 3. As a consequence, the central axis of this hinge (or more correct, bisector of the angle $\alpha$ ) is displaced only for at about $50^{\circ}$ with respect to the initial position, while residual $130^{\circ}$, to complement the angle of the overall swivel of the door, equal to $180^{\circ}$, is completed by rotation of the door leaf with respect to the line of bisector. Anyway, the implementation in the hinge, described in Liermann [6], the curved sliding pathway has the aim to make the frame part smaller and does not considered as a mean of changing the trajectory.

In Liermann [6] is presented the the type of concealed hinge which allows the closing the door with the sealing of the external and internal edges of the door leaf simultaneously. This sealing is extremely important from the point of view of an eco-friendly home design for energy conservation. This hinge consists substantially from the two elements, one is the hinge with two axes, and another is an element which must determine the unique trajectory of the door movement. But the position this last element is practically undefined, which result in jamming. So, this configuration of hinge is not capable of working successfully. In contrary, the mechanism of the hinge with five axes can be created in the manner at which the door is closed with the sealing the both edges of the door leaf and at the same time allows door opening up to $180^{\circ}$.

In the recent time in the advanced architectural solutions of internal decor design were used the doors with invisible hinges. It makes elegant, secure and allows different designer's solutions like, for example, the following: the doors can be set with the plain of the face of the wall flush with the plane of the face of the door. All recently known invisible hinges having 5 axes, which allow opening the door up to $180^{\circ}$, are constructed almost empirically without any clear knowledge of the kinematic behavior of hinge.

\section{Conclusions}

In this article it was found the geometrical layout, which clearly represents all possible hinge configurations. It was determine the principal parameters, responsible for hinge and door movements that are the angle between brackets, the lengths of brackets and sliding guides. In particular, it was defined the sliding guides pathways, which provide the low, moderate and high rotation of the door at the same value of decrement of the angle between brackets. We have delimited the area, in which the sliding guides can exist geometrically.

Further we have derived the equations to obtain the angle of door rotation for three different sliding pathways, "low", "moderate" and "high rotation", as a function of initial angle between brackets and ratios of brackets lengths for the door part of the hinge and for the frame part. By using these equations we analysed the variation of the angle of the door leaf opening as a function of brackets lengths ratio " $k$ ", and as a function of the angle between brackets " $\alpha$ ". It was shown that:

1. The angle of door opening reach it maximum value at the brackets lengths ratio $\mathrm{R} / \mathrm{r}=3 / 2$.

2. The derivative of angle of door opening with respect to " $\alpha$ " (that 
is: $d(\beta+\mu) / d \alpha)$ is significantly high at low " $\alpha$ " and diminished when " $\alpha$ " is rise. In another word, when " $\alpha$ " decrements, for example, from $10^{\circ}$ to $0^{\circ}$, the door is opened for higher angle, than when " $\alpha$ " goes from $30^{\circ}$ to $20^{\circ}$.

3. The position and trajectory of sliding guides have a strong influence on the door movement: if the trajectory, as defined above, is of "low rotation", the changing in the angle " $\alpha$ " from $80^{\circ}$ to $0^{\circ}$ can produces only $50^{\circ}$ of the angle of door opening, but the same decrement of the angle " $\alpha$ " can result in complete revolution of the door (up to $360^{\circ}$ ) if both trajectories of sliding guides (the door part and the frame part) are of "high rotation".

Also, the presented kinematic analysis has been verified by comparison with real model, created by using the CAD model. It was found a good conformity between the CAD model and analytical calculations. The analysis was applied to examination of the known configurations of the hinge and in these solutions, was found some shortcomings.

Finally, it's must be emphasized, that this approach represents an effective tool for creation the desirable trajectory of the movement of invisible hinge, because up to now to create any trajectory, different from linear symmetrical sliding guides, originally designed by Joseph Soss, the authors go in blind,without clear understanding the hinge kinematics, as can be seen from the above cited patents $[2-7,9,10]$.

\section{Acknowledgments}

The authors are grateful to Dr. Luca De Robertis e Mr. Roberto Franco, which help to obtain the experimental results and to construct the new types of invisible hinges.

\section{References}

1. Schmidt HF (1974) Invisible hinge. Patent and Trademark Office, Washington DC, USA.

2. Caldari G (2002) Hidden hinge. US Patent 6487755 B1, Patent and Trademark Office, Washington DC, USA.

3. Neukoetter H, Liermann N (2012) Concealed door hinge, US Patent 8196256 B2. U.S. Patent and Trademark Office, Washington DC, USA.

4. Neukoetter H (2013) Door hinge for recessed installation between door edge and jamb. US Patent. U.S. Patent and Trademark Office, Washington, DC, USA.

5. Way WR (1937) Closure for wall openings. US Patent 2100928. U.S. Patent and Trademark Office, Washington DC, USA.

6. Liermann $\mathrm{N}$ (2012) Door hinge for hidden placement between door frame and door leaf, and door provided with the door hinge. US Patent 2012/0180396 A1. Washington, DC: U.S. Patent and Trademark Office, Washington, DC, USA.

7. Liermann $N(2007)$ Door hinge for hidden assembly between door frame and door wing. European Patent No. EP 1754848 A2, European Patent Office, Munich.

8. Neukötter H (2009) Hinge: European Patent No. EP 2130997 A2, European Patent Office, Munich.

9. Robinson $\mathrm{H}$ (2014) How to build an environmentally friendly, energy efficient home.

10. Soss J (1924) Hinge: U.S. Patent and Trademark Office, Washington, DC, USA. 